\title{
Polaronic signatures in the optical properties of the electron-doped cuprate superconductor $\mathrm{Nd}_{2-x} \mathrm{Ce}_{x} \mathrm{CuO}_{4}$
}

\author{
E. Cappelluti, ${ }^{1,2}$ S. Ciuchi, ${ }^{3,1}$ and S. Fratini ${ }^{4,5}$ \\ ${ }^{1}$ SMC Research Center and ISC, INFM-CNR, v. dei Taurini 19, 00185 Rome, Italy \\ ${ }^{2}$ Dipartimento di Fisica, Università "La Sapienza," P.le A. Moro 2, 00185 Rome, Italy \\ ${ }^{3}$ Dipartimento di Fisica and CNISM, Università dell'Aquila, via Vetoio, I-67010 Coppito-L'Aquila, Italy \\ ${ }^{4}$ Institut Néel-CNRS, Université Joseph Fourier, BP 166, F-38042 Grenoble Cedex 9, France \\ ${ }^{5}$ Instituto de Ciencia de Materiales de Madrid (CSIC), Sor Juana Inés de la Cruz 3, E-28049 Madrid, Spain
}

(Received 18 November 2008; published 14 January 2009)

\begin{abstract}
We investigate the temperature and doping dependences of the midinfrared band in the optical conductivity of electron-doped cuprates in terms of magnetic/lattice polaron formation. We employ dynamical mean-field theory in the context of the Holstein- $t-J$ model for the specific case of $\mathrm{Nd}_{2-x} \mathrm{Ce}_{x} \mathrm{CuO}_{4}$. We find that the onset of short-range magnetic correlations at the pseudogap temperature triggers the formation of the lattice polaron responsible for the midinfrared band.
\end{abstract}

DOI: 10.1103/PhysRevB.79.012502

PACS number(s): 74.25.Gz, 71.10.Fd, 71.38.-k, 78.20.Bh

Understanding the phase diagram of high- $T_{c}$ superconductors still represents, 20 years after their discovery, one of the main challenges in this field. One of the reasons for such difficulties relies on the fact that different interactions are simultaneously operating, involving different degrees of freedom. On one hand, evidence is gathering, from different experimental techniques, about a relevant role of the electron-lattice interaction. ${ }^{1-5}$ On the other hand, short-range antiferromagnetic (AF) correlations, ruled by the exchange coupling $J$, are still present in the normal state even when true long-range AF ordering is destroyed. The interplay between these two kinds of interactions has been recently pointed out in undoped and weakly doped cuprates, where angle-resolved photoemission spectroscopy (ARPES) revealed a broad high-energy peak well distinguished from the quasiparticle states with vanishingly small spectral weight. ${ }^{6,7}$ The broadness of this peak has been interpreted in terms of a multiphonon Franck-Condon structure typical of a lattice polaron and superposed on the dynamics of a single hole in the $t$ - $J$ model ${ }^{6-10}$ The validity of this scenario at finite doping and its relation with the pseudogap observed by means of different techniques is however, not clear.

Among the different families of copper oxides, electrondoped cuprates represent the most suitable systems in which to investigate the interplay between magnetic and lattice polarons. This is because, unlike hole-doped systems, the longrange $\mathrm{AF}$ ordering extends up to quite large doping levels $\delta \approx 0.14$, so that the relative importance of long-range vs short-range magnetic correlation can be more clearly addressed. The optical conductivity (OC) of the electron-doped compound $\mathrm{Nd}_{2-x} \mathrm{Ce}_{x} \mathrm{CuO}_{4}$ (NCCO) has been systematically analyzed as a function of temperature and doping in Ref. 11. There, a midinfrared (MIR) shoulder associated with a pseudogap state has been observed in the spectra at finite doping. Quite interestingly, they observed (i) a shift of the MIR peak toward lower frequencies upon increasing $\delta$, corresponding to a pseudogap closing with doping; (ii) a filling of the pseudogap upon increasing the temperature; and (iii) the absence of any signature of the Néel temperature in the temperature evolution of the pseudogap spectral weight (de- fined as the spectral weight in the region between the MIR shoulder and a low-energy infrared cutoff $\omega_{\min } \simeq 0.12 \mathrm{eV}$ ), whereas a remarkable nonmonotonic kink in the temperature evolution of the pseudogap spectral weight is present at a higher temperature $T^{*}$. The latter feature suggests a prominent role of short-range correlations in governing the observed spectral properties.

A detailed theoretical study of the polaron features in the optical conductivity of one hole in the Holstein- $t-J$ model, using dynamical mean-field theory (DMFT), has been recently reported in Ref. 12. In this Brief Report, we apply this approach to analyze the optical data in electron-doped cuprates NCCO where exhaustive measurements as a function of both temperature and doping are available. ${ }^{11}$ We show that the MIR band of the optical conductivity in these compounds can be naturally interpreted in terms of spin/lattice polarons resulting from the joint positive cooperation between the electron-phonon (el-ph) and magnetic exchange interactions. ${ }^{12,13}$ In this framework we are able to explain the existence of a pseudogap in the optical spectra, as well as its evolution with doping and temperature. From the comparison between theory and experiments we estimate a value for the local el-ph coupling on the order of $\lambda \approx 0.7$, in very good agreement with a recent analysis of ARPES measurements. ${ }^{14}$

We now examine the above scenario in the light of the theoretical DMFT results. We consider the motion of one electron added to the half-filled antiferromagnetic background and interacting à la Holstein with local dispersionless phonons. Due to the particle-hole symmetry on the parent Hubbard-Holstein Hamiltonian, this problem is formally equivalent to considering one hole in the Holstein- $t-J$ model. Using the linear spin-wave approximation, we can thus write the Hamiltonian as ${ }^{12,13}$

$$
\begin{aligned}
H= & \frac{t}{2 \sqrt{z}} \sum_{\langle i j\rangle}\left(h_{j}^{\dagger} h_{i} a_{j}+\text { H.c. }\right)+\frac{\widetilde{J}}{2} \sum_{i} a_{i}^{\dagger} a_{i} \\
& +g \sum_{i} h_{i}^{\dagger} h_{i}\left(b_{i}+b_{i}^{\dagger}\right)+\omega_{0} \sum_{i} b_{i}^{\dagger} b_{i},
\end{aligned}
$$

where $z$ is the coordination number, $a^{\dagger}$ and $b^{\dagger}$ are the cre- 
ation operators for boson spin defects and phonons, respectively, and $h^{\dagger}$ is the single spinless charge operator. A local el-ph coupling constant $\lambda=g^{2} / \omega_{0} t$ can be defined as the polaron energy in units of the half-bandwidth $t$. The parameter $\widetilde{J}$ is an effective exchange interaction. It is linked to the microscopic $J$ by the relation $\widetilde{J}=J m$, where $m$ is the average on-site magnetization which, in the $z \gg 1$ limit, is governed by the Curie-Weiss equation $m=\tanh (\beta J m / 4)$ with Néel temperature $T_{N}=J / 4$. The one-particle Hamiltonian (1) can be thought to be a representative also of the finite doping case as long as we are in the dilute limit, namely, as long as the interparticle distance $d_{\mathrm{P}-\mathrm{P}}$ is larger than the spin polaron size $L_{p}$. For $\delta \leqq 0.14$ this implies $L_{p} \lesssim 2.7$ in units of the lattice constant. We shall discuss later the validity of this condition. A further implication of the finite doping regime is that the effective exchange energy $\widetilde{J}$ should be considered as renormalized by finite charge-density effects and is thus doping dependent, $\widetilde{J}=\widetilde{J}(\delta)$.

The Holstein- $t$ - $J$ Hamiltonian (1) is the basis for discussing the physics of a spin/lattice polaron, namely, a traveling charge carrying along an on-site phonon cloud and surrounded by a local destruction of the AF background of size $L_{p}$. Different properties of this object can be investigated and, as a general rule, the choice of a particular theoretical approach depends on which property is under examination and on its feasibility to investigate it. Quasiparticle dispersion, its relative spectral weight, and effective mass, for instance, are intrinsically related to the coherent motion of the polaron as a whole. On the other hand, the internal local structure of the spin/lattice polaron, responsible for the optical absorption at finite frequency, is mainly related to incoherent processes which can be safely caught by the dynamical mean-field approach, where coherent motion is disregarded. ${ }^{12,13}$

One of the main intriguing characteristics of the spin/ lattice polaron that can be captured by DMFT within the context of the Holstein- $t-J$ model ${ }^{15}$ is that the electronphonon and the exchange interaction act in a cooperative way to reduce the local hopping amplitude and hence to establish polaronic features. . $^{12,13,16-20}$ An interesting consequence of this scenario is that lattice polaron features can be in principle switched on and off by varying the magnetic exchange coupling $\widetilde{J}$ at given el-ph coupling $\lambda$. Our analysis shows that this is precisely what occurs in NCCO. On the physical ground, the effective exchange coupling depends on the charge doping $\delta$ and on the temperature $T$, in such a way that increasing $\delta$ and $T$ leads to a reduction in $\widetilde{J}$ and hence to an increase in the spin polaron size. In this context we interpret the pseudogap temperature $T^{*}$ as the highest temperature where the spin polaron size $L_{p}$ is smaller than the AF correlation length $\xi_{\mathrm{AF}}$. For $T>T^{*}\left(L_{p}>\xi_{\mathrm{AF}}\right)$ the charge motion does not probe anymore the AF environment and a paramagnetic analysis with $\widetilde{J}=m=0$ is enforced. We can identify thus the experimental $T^{*}$ with the Néel temperature $T_{N}$ of the DMFT solution of the Holstein- $t-J$ model, where the AF correlation length jumps from $\xi_{\mathrm{AF}}=0$ for $T>T_{N}$ to $\xi_{\mathrm{AF}}=\infty$ for $T \leq T_{N}$ and the spin polaron size from $L_{p}=\infty$ for $T>T_{N}$ to a finite value for $T \leq T_{N}$. Along this perspective we can deter-
TABLE I. Doping evolution of experimental data and theoretical parameters as a function of doping $\delta . T^{*}$ is the pseudogap temperature taken from Ref. 11, $J$ is the theoretical estimate of lowtemperature exchange coupling corresponding to $T^{*}, L_{p}$ is the average number of spin defects, i.e., the spin polaron size in units of the lattice constant, and $d_{\mathrm{P}-\mathrm{P}}$ is the average particle-particle distance given by $d_{\mathrm{P}-\mathrm{P}}=1 / \sqrt{\delta}$, also in units of the lattice constant.

\begin{tabular}{lcccc}
\hline \hline$\delta$ & 0.05 & 0.075 & 0.1 & 0.125 \\
$T^{*}(\mathrm{~K})$ & 440 & 340 & 300 & 200 \\
$J(\mathrm{meV})$ & 152 & 117 & 103 & 69 \\
$L_{p}$ & 0.14 & 0.19 & 0.27 & 1.01 \\
$d_{\text {P-P }}$ & 4.5 & 3.7 & 3.2 & 2.8 \\
\hline \hline
\end{tabular}

mine the (doping-dependent) low-temperature effective exchange energy $\widetilde{J}(T=0)=J$, where $m=1$, from the experimental temperature $T^{*}=T_{N}=J / 4$. The estimates obtained as a function of $\delta$, in good agreement with experimental estimates, ${ }^{21}$ are collected in Table I along with other characteristic quantities. The temperature dependence of the magnetization can thus be evaluated from the Curie-Weiss relation. From photoemission spectroscopy we take a halfbandwidth value $t=1 \mathrm{eV}$ for $\mathrm{NCCO},{ }^{22-24}$ and we set also $\omega_{0}=70 \mathrm{meV}$, consistent with the energy window of the optical phonons in undoped cuprates. With these values, the only free adjustable parameter is the electron-phonon coupling constant $\lambda$. We are going to show that the overall experimental scenario ${ }^{11}$ is naturally reproduced within the context of a spin/lattice polaron by assuming a dopingindependent el-ph coupling $\lambda=0.7$. We would stress that this does not rule out a possible weak dependence of $\lambda$ on doping which would provide an even stronger agreement with the experimental data.

It should be stressed that an el-ph coupling $\lambda=0.7$, at $\omega_{0} / t=0.07$, is not sufficient to induce lattice polaron effects in the absence of exchange coupling since a critical value $\lambda_{c}=0.91$ is needed to establish a lattice polaron state at $J$ $=0 .{ }^{25}$ This means that lattice polaron features in these compounds can be found only in the presence of (short-range) AF correlations and they are effectively tuned by the dopingdependent exchange coupling $J(\delta)$. In order to show and quantify this issue we plot in Fig. 1 the renormalized phonon probability distribution function $P(n)\left[\Sigma_{n} P(n)=1\right]$ for different values of the exchange coupling spanning the experimen-

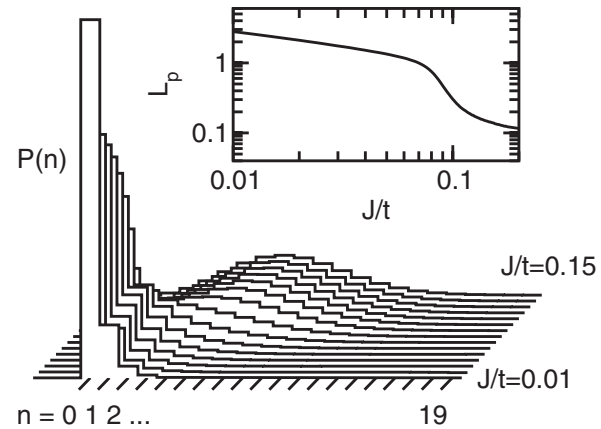

FIG. 1. Phonon distribution function $P(n)$ (main panel) and spin polaron size $L_{p}$ (inset) as a function of exchange coupling. 


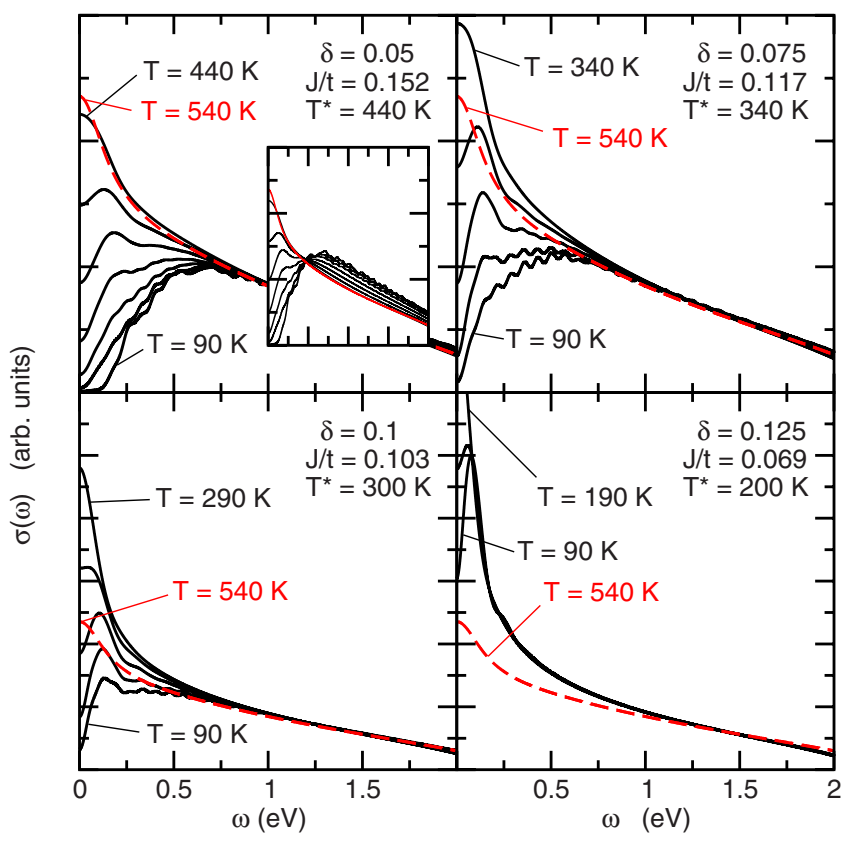

FIG. 2. (Color online) Temperature evolution of the optical conductivity $\sigma(\omega)$ for different exchange couplings (dopings) and for $T \leq T^{*}: T=90,140,190,240,290,340,390,440 \mathrm{~K}$. Also shown is the optical conductivity at $T=540 \mathrm{~K}$ in the normal state (dashed red line). Inset: optical conductivity for $\delta=0.05$ normalized by its total spectral weight.

tal range of $J$ (see Table I). The magnetic-induced lattice polaron crossover occurs for $J / t \simeq 0.083(J=83 \mathrm{meV}$ in energy units), where $P(n)$ changes abruptly from a distribution peaked at $n=0$ to a multiphonon broad structure at finite $n$ typical of a polaronic lattice distortion. The strong interplay between lattice and magnetic degrees of freedom is also witnessed by the dependence of the spin polaron size $L_{p}$ on $J$ at $T=0$ obtained by the Hellmann-Feynman theorem, ${ }^{13}$ as shown in the inset of Fig. 1. Here the onset of the lattice polaron for $J / t \gtrsim 0.083$ leads to a further self-trapping localization of the charge and to an abrupt drop of $L_{p}$ in the polaronic regime. Comparing the typical values of the exchange energy estimated from the experimental data reported in Table I, we can predict lattice polaron features, accompanied by a very small amount of spin defects, for dopings $\delta$ $=0.05,0.075,0.1$, while at the larger doping $\delta=0.125 \mathrm{spin} /$ lattice, polaron features are already remarkably reduced. It is also comforting to check that for all the dopings considered in the present analysis $\delta=0.05-0.125$, both on the polaronic and on the nonpolaronic side, the estimate of the spin polaron size $L_{p}$ is always smaller than the average particleparticle distance $d_{\text {P-P }}$ (see Table I), confirming the validity of the present one-particle analysis in the dilute limit.

Let us now discuss the effects of the magnetic-induced lattice polaron formation on the optical conductivity. In Fig. 2 we plot $\sigma(\omega)$ evaluated for the different exchange couplings corresponding to different doping levels (see Table I). We should stress once more that, due to the local approximation enforced by the $z=\infty$ DMFT limit, only incoherent contributions to the $\mathrm{OC}$ are here taken into account, so that no Drude-type peak is present. Disregarding the coherent
Drude peak, the resemblance with the experimental data of Ref. 11 is remarkable. In particular we observe at low temperature a broad incoherent shoulder in the MIR energy range which characterizes the pseudogap regime. This feature represents the natural counterpart, in the optical data, of the broad multiphonon structure responsible for the incoherent peak observed in ARPES and is a natural signature of the onset of the lattice polaron. Consistent with this picture, the energy scale of this broad MIR band is reduced as polaronic features are weakened upon doping (Fig. 2), which causes a closing of the optical pseudogap. Also interesting is the role of temperature also reported in Fig. 2. While the magnitude of the exchange coupling $J$ rules the energy scale of the MIR band $^{12}$ at low temperature, and hence drives the closing of the pseudogap with $\delta$, increasing the temperature for $T<T^{*}$ leads to a filling of the pseudogap itself (see in particular the upper panels in Fig. 2), in good agreement with Ref. 11. Eventually for $T>T^{*}$ there is a considerable smoothing of the OC features with respect to the spectra at $T \approx T^{*}$.

This temperature evolution can be nicely traced by evaluating the "MIR optical spectral weight" $W_{\text {MIR }}$, which was defined in Ref. 11 as the optical spectral weight between a low-energy cutoff at $\omega_{\text {low }}=0.12 \mathrm{eV}$ and a high-energy isosbestic point. Quite notably, the temperature evolution of the spectral weight does not show any signature of the AF longrange order at the Néel temperature $T_{N}^{\exp }$, whereas a nonmonotonic kink in the temperature evolution at a higher temperature $T^{*}$ is clearly visible. This observation can be naturally explained within the present (local) polaronic approach since the local polaron processes responsible for the incoherent shoulder in Fig. 2 cannot distinguish between short-range and long-range AF orderings as long as $L_{p}$ $<\xi_{\mathrm{AF}}$. For the same reasons, anomalies in $W_{\mathrm{MIR}}$ could be expected at $T \gtrsim T^{*}$ ( $T \gtrsim T_{N}$ in our analysis), where $L_{p} \gtrsim \xi_{\mathrm{AF}}$ and the concept itself of a spin polaron breaks down. In order to quantify this issue, following Ref. 11 , we define $W_{\text {MIR }}$ as the optical spectral weight between $\omega_{\text {low }}=0.12 \mathrm{eV}$ and an isosbestic point at higher energy. Such an isosbestic point is not apparently evident in the main panels of Fig. 2. It should be considered that, however, interband transitions, contributing to a $\omega$-dependence background, are not taken into account within a single-band model, violating the $f$ sum rule. Experimental data of Ref. 11 on the other hand show that this $f$ sum rule is fulfilled already in the midenergy window $\omega$ $\in[0.12,1.4] \mathrm{eV}$. At the lowest order we can enforce this information by requiring that even our single-band results fulfill a temperature-independent sum rule in this energy range. This simple procedure gives rise in a natural way to an isosbestic point, as shown in the inset of Fig. 2 for the specific case of $\delta=0.05$ and found for any other value of $\delta$. The temperature evolution of $W_{\mathrm{MIR}}$ is shown in Fig. 3, where we plot $\Delta W_{\mathrm{MIR}}=W_{\mathrm{MIR}}(T=540 \mathrm{~K})-W_{\mathrm{MIR}}(T)$ to have a direct comparison with Ref. 11. The resemblance with the experimental data is striking. For all dopings we find that the reduction in $\Delta W_{\mathrm{MIR}}$ as a function of $T$, which reflects the filling of the pseudogap, is accompanied by a change in behavior at $T^{*}$. We associate these behaviors with two different regimes. In the high-temperature regime $T>T^{*}$ polaronic effects are not operative. Increasing the temperature leads to the usual smearing of the overall shape of the optical spectra and thus 


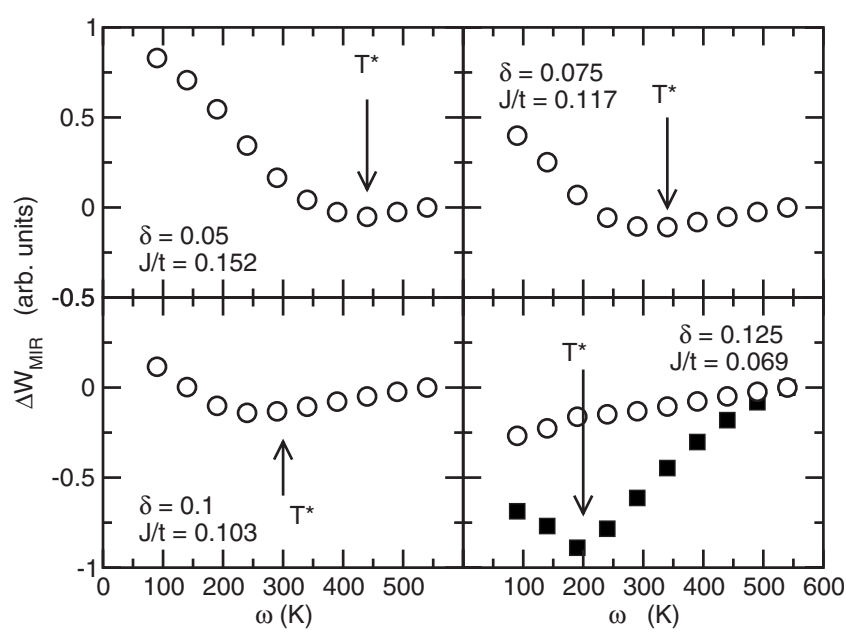

FIG. 3. Temperature dependence of $\Delta W_{\text {MIR }}$ for different exchange couplings corresponding to different doping levels. Empty circles and black squares are obtained by using a lower cutoff $\omega_{\text {low }}=0.12 \mathrm{eV}$ and $\omega_{\text {low }}=0 \mathrm{eV}$, respectively.

to a depletion of the spectral weight in the MIR energy window (i.e., an increase in $\Delta W_{\mathrm{MIR}}$ ). In the polaronic regime at $T<T^{*}$ this effect is counteracted by the stronger temperature dependence of the polaronic effects. The latter leads to a filling of the pseudogap and therefore to an increase in $W_{\text {MIR }}$ (a decrease in $\Delta W_{\text {MIR }}$ ) upon increasing the temperature. The competition between these two effects gives rise to a kink in the temperature evolution of $\Delta W_{\text {MIR }}$. This kink is not clearly visible at the highest doping $\delta=0.125$ in Fig. 3 . This is due to the fact that, in contrast to Ref. 11, in our data at $\delta=0.125$ the weak polaronic MIR peak is at $\omega \approx 0.08 \mathrm{eV}$, which is out of the range $[0.12,1] \mathrm{eV}$ of the spectral weight integration. The agreement with Ref. 11 is recovered by extending the integration of $\Delta W_{\mathrm{MIR}}$ down to $\omega_{\text {low }}=0$ (black squares in the last panel of Fig. 3).

In conclusion, in this Brief Report we have analyzed the optical data of the electron-doped cuprate $\mathrm{Nd}_{2-x} \mathrm{Ce}_{x} \mathrm{CuO}_{4}$ as a function of doping and temperature by means of the DMFT results for one electron in the Holstein- $t-J$ model. We have shown that the origin of the MIR band, the optical pseudogap, as well as its doping and temperature dependences can be naturally explained. This can be done in terms of a polaronic scenario; where antiferromagnetic correlations tune the formation of the lattice polaron, which is responsible for the MIR absorption edge. This finding is in contrast to a recent publication where a doping-dependent el-ph coupling is assumed. ${ }^{26}$ From our analysis we estimate an el-ph coupling on the order of $\lambda \simeq 0.7$. This value essentially agrees with that recently obtained from an analysis of ARPES measurements in the same class of materials. ${ }^{14}$ Our results illustrate the relevance of the positive interplay between lattice and spin degrees of freedom in electron-doped cuprates, where the AF ordering extends over a large doping window, making these compounds ideal systems where one can investigate such effects.

We thank Y. Onose, Y. Tokura, A. S. Mishchenko, V. Cataudella, and G. De Filippis for useful discussions. We also thank Y. Onose and Y. Tokura for having made available their experimental data. E.C. and S.C. acknowledge also financial support from MIUR under the Research Program MIUR-PRIN 2005.
${ }^{1}$ A. Lanzara et al., Nature (London) 412, 510 (2001).

${ }^{2}$ G.-H. Gweon et al., Nature (London) 430, 187 (2004).

${ }^{3}$ R. Khasanov et al., J. Phys.: Condens. Matter 16, S4439 (2004).

${ }^{4}$ R. Khasanov et al., Phys. Rev. Lett. 92, 057602 (2004).

${ }^{5}$ J. Lee et al., Nature (London) 442, 546 (2006).

${ }^{6}$ K. M. Shen et al., Phys. Rev. Lett. 93, 267002 (2004).

${ }^{7}$ K. M. Shen et al., Phys. Rev. B 75, 075115 (2007).

${ }^{8}$ A. S. Mishchenko and N. Nagaosa, Phys. Rev. Lett. 93, 036402 (2004).

${ }^{9}$ A. S. Mishchenko and N. Nagaosa, Phys. Rev. B 73, 092502 (2006).

${ }^{10}$ V. Cataudella et al., Phys. Rev. Lett. 99, 226402 (2007).

${ }^{11}$ Y. Onose et al., Phys. Rev. B 69, 024504 (2004).

${ }^{12}$ E. Cappelluti et al., Phys. Rev. B 76, 125111 (2007).

${ }^{13}$ E. Cappelluti and S. Ciuchi, Phys. Rev. B 66, 165102 (2002).

${ }^{14}$ S. R. Park et al., Phys. Rev. Lett. 101, 117006 (2008).

${ }^{15}$ Things can be different when a second-nearest hopping term $t^{\prime}$ is considered; see G. De Filippis et al., Phys. Rev. Lett. 99,
146405 (2007).

${ }^{16}$ J. Zhong and H. B. Schuttler, Phys. Rev. Lett. 69, 1600 (1992).

${ }^{17}$ A. Ramšak et al., Phys. Rev. B 46, 14305 (1992).

${ }^{18}$ H. Fehske et al., Phys. Rev. B 51, 16582 (1995).

${ }^{19}$ P. Prelovšek et al., Phys. Rev. Lett. 96, 086402 (2006).

${ }^{20}$ L. Vidmar et al., arXiv:0812.2131 (unpublished).

${ }^{21}$ R. J. Birgenau and G. Shirane, in Physical Properties of High Temperature Superconductors, edited by D. M. Ginsberg (World Scientific, Singapore, 1989), Vol. 1.

${ }^{22}$ N. P. Armitage et al., Phys. Rev. Lett. 88, 257001 (2002); N. P. Armitage et al., Phys. Rev. B 68, 064517 (2003).

${ }^{23}$ R. S. Markiewicz et al., Phys. Rev. B 72, 054519 (2005).

${ }^{24}$ M. Ikeda et al., J. Supercond. Novel Magn. 20, 563 (2007).

${ }^{25}$ We define in an unambiguous way a lattice polaron instability, for generic $\lambda$ and $J$, by the condition $P(n=0) \leq P(n \neq 0)$, where $P(n)$ is the phonon distribution function (see Ref. 13).

${ }^{26}$ A. S. Mishchenko et al., Phys. Rev. Lett. 100, 166401 (2008). 